\title{
A Taylor series approach for coupled queueing systems with intermediate load
}

\author{
Ekaterina Evdokimova, Sabine Wittevrongel and Dieter Fiems \\ Department of Telecommunications and Information Processing, Ghent University \\ St-Pietersnieuwstraat 41, 9000 Gent, Belgium
}

\begin{abstract}
We focus on the numerical analysis of a coupled queueing system with Poisson arrivals and exponentially distributed service times. Such a system consists of multiple queues served by a single server. Service is synchronised meaning that there is a departure from every queue upon service completion and there is no service whenever one of the queues is empty. It was shown before that the terms in the Maclaurin series expansion of the steady state distribution of this queueing system when the service rate is sent to 0 (overload) can be calculated efficiently. In the present paper we extent this approach to lower loads. We focus on a sequence of Taylor series expansions of the stationary distribution around increasing service rates. For each series expansion, we use Jacobi iteration to calculate the terms in the series expansion where the initial solution is the approximation found by the preceding series expansion. As the generator matrix of the queueing system at hand is sparse, the numerical complexity of a single Jacobi iteration is $O(N M K)$, where $N$ is the order of the series expansion, $K$ is the number of queues and $M$ is the size of the state space. Having a good initial solution reduces the number of Jacobi iterations considerably, meaning that we can find a sequence of good approximations of the steady state probabilities fast.
\end{abstract}

\section{INTRODUCTION}

Motivated by kitting processes in assembly systems, we study a queueing system with coupled queues. Kitting is the process of collecting all parts in a kit prior to assembly [1]. As a strategy for supplying materials at the assembly line, delivering complete kits rather than containers of equal parts mitigates storage space requirements at the assembly line and may lead to overall production cost reductions. However, additional space and time needs to be allocated for the preparation of the kits.

A coupled queueing system consists of multiple queues, which are served simultaneously. That is, when all queues are non-empty, all customers that are at the head of the line of their queue are served simultaneously and depart when the service is completed. However, when one of the queues is empty, no customers are served at all. In terms of the kitting process discussed above, service corresponds to collecting all parts in the kit, the parts being the customers in the queues and the queues representing the part inventories. Whenever one of the queues is empty, a part is missing and the kit cannot be completed.

We study the coupled queueing system assuming Poisson arrivals in all queues and assuming exponentially distributed service times. Even with these "Markovian" assumptions, the analysis of the coupled queueing system is challenging. First, one cannot assume that queues have infinite capacity as the resulting Markov process is null recurrent, see [5] for the coupled queueing system with only two queues. Secondly, for a $K$ queues with capacity $C$ the state space of the Markov chain is $(C+1)^{K}$ such that a direct solution of the Markov chain is not numerically feasible for moderate $C$ and $K$. Finally, matrix-analytic methods for neither $M / G / 1$-type nor $G / M / 1$-type queueing systems apply. To overcome these challenges, literature proposes two alternative approaches. The first approach aims at decomposing the queueing system into a number of independent queueing systems [2]. Such an analysis approximates the interaction between the different queues by a simpler process which facilitates the analysis. Alternatively, in $[3,4]$ the Markov process is studied without decomposition. In this paper it is shown that the terms of the Maclaurin series expansion of the steady-state distribution in the service rate can be obtained at low computational cost. The series expansion of the performance measures can then be easily obtained from the calculated steady-state distribution. However, the numerical approach advocated there only leads to results when the service rate is close to 0 , or equivalently, when the 
load of the system is high.

The present contribution builds on the results of [3] and [4], but considers the system when the load is lower. Balancing computational cost and accuracy, we investigate the use of Taylor series expansions to calculate the performance measures for a wider range of the service rates. In contrast to [3, 4], the series expansion around some service rate $\mu_{0} \neq 0$ cannot be obtained directly. Therefore we rely on iterative solution methods - in particular on Jacobi iteration - to solve for the terms in the series expansion. For such iterative methods, a good initial guess of the solution can reduce the number of required iterations considerably. Therefore, we here consider a sequence of Taylor series expansions around increasing values of the service rate. As initial guess for solving for the terms in these series expansions, we use the approximation found in the preceding expansion. The numerical method is sketched in the next section. We then illustrate our approach by a numerical example prior to drawing conclusions.

\section{PERFORMANCE ANALYSIS}

$$
\begin{aligned}
& \pi\left(i_{1}, i_{2}, \ldots, i_{K}\right)\left(\mu \prod_{\ell=1}^{K} \mathbf{1}_{i_{\ell}>0}+\sum_{\ell=1}^{K} \mathbf{1}_{i_{\ell}<C_{\ell}} \lambda_{\ell}\right)= \\
& \pi\left(i_{1}+1, i_{2}+1, \ldots, i_{K}+1\right) \mu \prod_{\ell=1}^{K} \mathbf{1}_{i_{\ell}<C_{\ell}}+\sum_{\ell=1}^{K} \pi\left(i_{1}, \ldots, i_{\ell-1}, i_{\ell-1}, i_{\ell+1}, \ldots, i_{K}\right) \lambda_{\ell}, \\
& \sum_{n=0}^{\infty} \pi\left(i_{1}, i_{2}, \ldots, i_{K}\right) \mu^{n}\left(\mu \prod_{\ell=1}^{K} \mathbf{1}_{i_{\ell}>0}+\sum_{\ell=1}^{K} \mathbf{1}_{i_{\ell}<C_{\ell}} \lambda_{\ell}\right)= \\
& \sum_{n=0}^{\inf } \pi\left(i_{1}+1, i_{2}+1, \ldots, i_{K}+1\right) \mu^{n+1} \prod_{\ell=1}^{K} \mathbf{1}_{i_{\ell}<C_{\ell}}+\sum_{n=0}^{\infty} \sum_{\ell=1}^{K} \pi\left(i_{1}, \ldots, i_{\ell-1}, i_{\ell-1}, i_{\ell+1}, \ldots, i_{K}\right) \lambda_{\ell} \mu^{n} \mathbf{1}_{\left\{i_{\ell}>0\right\}},
\end{aligned}
$$

As it was mentioned above, here we consider a system with $K$ coupled queues with capacity $C$ served by a single server. The queues are being served simultaneously at the rate $\mu$ and only when all the queues have customers. Due to the structure of this Markov model, the generator matrix $Q^{(0)}$ for the case with no service $\mu=0$ is upper-triangular. Then the generator matrix in case $\mu \rightarrow 0$ can be represented as a perturbation of $Q^{(0)}$

$$
Q_{\mu}=Q^{(0)}+\mu Q^{(1)} .
$$

Then applying method from [3] we solve the balance equation for an overloaded system in terms of Maclaurin series expansion of a steady-state probability vector $\pi_{0}$. Thus, combining

$$
\pi_{0} Q_{\mu}=0 ; \pi_{0}=\sum_{i=0}^{\infty} \mu^{i} \pi_{0}^{(i)},
$$

the terms of $\pi_{0}$ can be found consecutively by identifying equal powers of $\mu$ in the balance equation

$$
\pi_{0}^{(0)} Q^{(0)}=0 ; \pi_{0}^{(n+1)} Q^{(0)}=-\pi_{0}^{(n)} Q^{(1)} .
$$

Since $Q^{(0)}$ has the triangular structure the complexity of the calculations does not exceed $O\left(M^{2}\right)$, where $M$ is a state space size. For the details of the calculation of the terms we refer the reader to the previous work [3].

Next let us consider a transition rate matrix given $\mu_{0} \neq 0$

$$
Q_{\mu}=Q^{(0)}+\mu_{0} Q^{(1)}+\mu Q^{(0)} .
$$

Unlike with $Q^{(0)}, Q_{\mu_{0}}=Q^{(0)}+\mu_{0} Q^{(1)}$ does not have triangular structure for $\mu_{0} \neq 0$, therefore, the technique above may not be exploited as for the overloaded regime. Therefore, looking for the solution around $\mu_{0}$ we further suggest the iterative Jacobi method for the numerical estimation of the Taylor expansion terms in the region of $\mu_{0}$

$$
\mu_{0}=\sum_{i=0}^{\infty}\left(\mu-\mu_{0}\right)^{i} \pi^{(i)}
$$


Thus, fist we calculate the solution in overload regime as $N$ terms $\pi_{0}=\left[\pi_{0}^{(0)}, \pi_{0}^{(1)}, \ldots \pi_{0}^{(N-1)}\right]$ that valid in the convergence region of $\mu \in\left[0 \ldots \mu_{0}\right]$, where $\mu_{0}$ is a boundary point of the convergence region of the initial solution. Then for the series expansion in region of $\mu \rightarrow \mu_{0}$ by analogy with (5) the terms can be calculated by solving

$$
\pi^{(0)} Q \mu_{0}=0 ; \pi^{(n+1)} Q_{\mu_{0}}=-\pi^{(n)} Q^{(1)} .
$$

Since $Q_{\mu_{0}}$ in case of extensive system might be too large for solving directly, we apply a Jacobi algorithm for the estimation of the terms. Therefore, we provide a solution for the system of linear equations $A \pi=\mathbf{b}$ in iterative manner

$$
\pi^{(n+1)}=D^{-1}\left(\mathbf{b}-R \pi^{(n)}\right)
$$

where $D$ is a diagonal matrix containing the elements of $A$ positioned on the main diagonal, and $R=A-D$. Having defined $A=Q_{\mu_{0}}$ and $b=-\pi^{(n)} Q^{(1)}$. In order to increase the efficiency of the Jucobi iterative algorithm the initial guess for the expansion terms $\hat{\pi}_{0}=\left[\hat{\pi}_{0}^{(0)}, \hat{\pi}_{0}^{(1)} \ldots \hat{\pi}_{0}^{(N)}\right]$ can be calculated using the terms of $\boldsymbol{\pi}_{0}$ by solving

$$
\pi_{0}(\mu)=\sum_{i=0}^{N} \mu^{i} \pi_{0}^{(i)}=\sum_{i=0}^{N-1} \hat{\pi}_{0}^{(i)}\left(\mu-\mu_{0}\right)^{(i)} .
$$

which results in the following expression for every term

$$
\hat{\pi}_{0}^{(j)}=\sum_{i=0}^{N-j} \frac{(i+j) !}{i ! j !} \pi_{0}^{(i+j)} \mu_{0}^{i} .
$$

\section{NUMERICAL RESULTS}

We exemplify the analysis by considering a paired queuing system containing 5 queues with capacity 10 which results in in a queueing model with state space size $M=161.051$. For the sake of demonstration the numerical estimations are compared with simulation samples. Figure 1 shows the results in terms of average system content $E[Q]$ as a function of service rate $\mu$, while the arrival rate at each queue is equal to $\lambda=1$. The plot includes a curve representing the initial solution, calculated by using $N=22$ terms of the Maclaurin series and valid in the region $\mu=[0 \ldots 0.7]$. Around the boundary value of the service rate $\mu_{0}=0.7$ the approximation starts deviating from the true values of the mean system load. Therefore, $\mu_{0}$ is considered further as a reference point for the Jukobi method to start the iterative estimation of the new Taylor expansion terms. The terms composing the initial solution are involved in the calculus as the initial guess. Observing the curves for various numbers of Jacobi iterations one can see that increasing number of iterations leads to the improving precision of the numerical result within the new convergence region prolonged till $\mu_{1}=0.85$. The difference between the boundaries $\Delta \mu=\mu_{1}-\mu_{0}$ characterizes the extension of the accuracy region that constitutes $21 \%$ of the original diapason.

The main result that we can observe on the Fig. 1 is that the suggested method allows for prolongation of the initial estimation beyond the diapason of high loads still preserving reasonable computational complexity $O(N M K)$, while methods for direct calculation of performance measures have computational cost of order $O\left(M^{3}\right)$.

\section{CONCLUSIONS}

In this work the method of analytical performance evaluation of systems with coupled queues is presented. Proposed approach can be used for planning and predicting the behaviour of kitting systems under the intermediate load. The outcome of the method is obtained as terms of Taylor series expansion allowing for the approximation of a steadystate distribution in the diapason of interest. After analysing the results of the previous research related to the coupled queues but focused on the overload regime, we made an assumption that by recalculating the series expansion at the boundary of the convergence region of the solution it is feasible to prolong the accuracy diapason. This initial hypothesis was supported by the results obtained in this study. For the given numerical example for system with 5 queues of capacity 10 the extension of accuracy region is $21 \%$. This observation demonstrates the ability of the proposed method to extend the results of the initial estimation beyond its convergence region. 


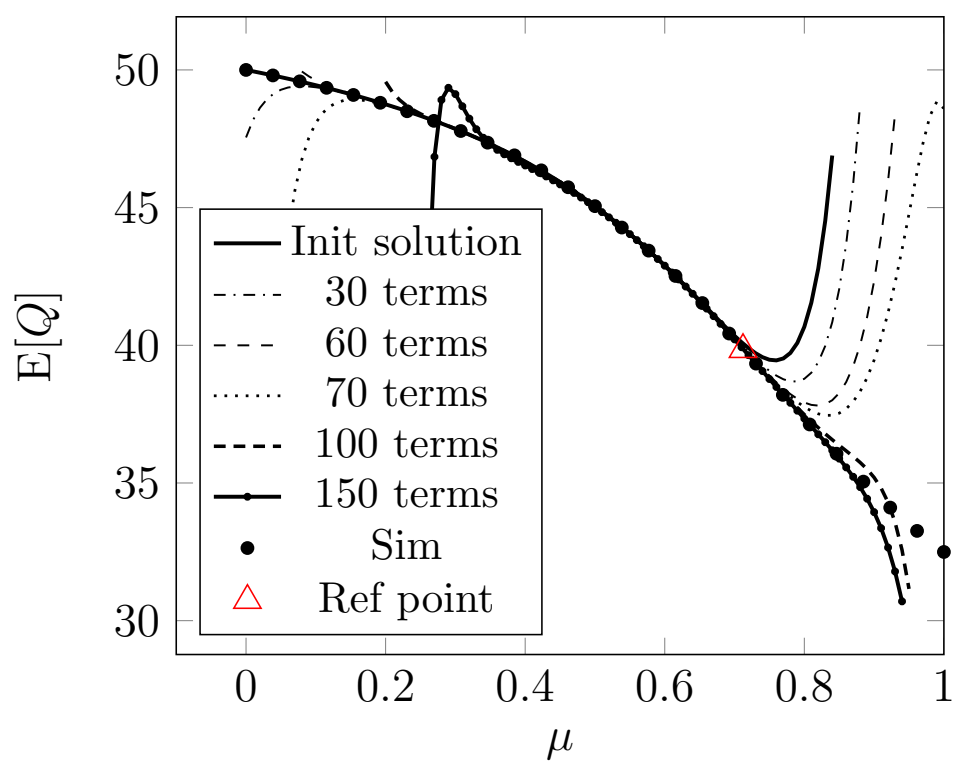

FIGURE 1. Mean buffer content for a paired queueing system with $K=5$ queues with capacity $C=10$.

The main contribution of this study is that the problem is tackled numerically, while the existent analysis of the large-scale queueing models mainly relies on the simulation. We can safely conclude that our performance analysis allows for the intermediate load performance evaluation. However, the region of accuracy may vary depending on the system size and structure and may not meet the requirements to the diapason where the performance estimations is requested. Therefore, the future research on this topic may study the opportunity of further recalculation of the Taylor series expansion for other loads. Additionally, it might be sensible to see what this approach can offer when analysing the large scale queueing models designed for other applications.

\section{ACKNOWLEDGMENTS}

\section{REFERENCES}

[1] S. Ramachandran, D. Delen. Performance analysis of a kitting process in stochastic assembly systems. Computers $\mathcal{F}$ Operations Research 32(3):449-463, 2005.

[2] W. Hopp, J.T. Simon. Bounds and heuristics for assembly-like queues. Queueing systems. 2(4):137-155, 1989.

[3] K. De Turck, E. De Cuypere, S. Wittevrongel, and D. Fiems. Algorithmic approach to series expansions around transient Markov chains with applications to paired queuing systems. In Proc. of the 6th International Conference on Performance Evaluation Methodologies and Tools, pp. 38-44, 2012.

[4] E. De Cuypere, K. De Turck, D. Fiems. A Maclaurin-series expansion approach to multiple paired queues. Operations Research Letters. 3(42):203-207, 2014.

[5] G. Latouche. Queues with paired customers. Journal of Applied Probability. 18(3):684-696, 1981. 